\title{
Toward collective animal neuroscience
}

Julia Sliwa

Science, 374 (6566), • DOI: 10.1126/science.abm3060

View the article online

https://www.science.org/doi/10.1126/science.abm3060

\section{Toward collective animal neuroscience

\author{
Social intelligence requires the study of both groups of brains and the \\ individual brain
}

\author{
By Julia Sliwa \\ Sorbonne Université, Paris Brain Institute-Institut du Cerveau, Inserm, CNRS, APHP, Hopital Pitié-Salpêtrière, Paris, France. Email: julia.sliwa@icm-
} institute.org

From humans to fish to ants, social animals coordinate their behavior when in groups, and their neural system might do so as well. Yet the individual brain, studied in isolation, has so far been the fundamental unit of analysis of most neuroscience research. Animal wisdom has been searched for in how neurons interact within individual brains. But what if embedding the brain in a group changed how it works? And even more boldly, what if the laws of wisdom were to be found in how brains interact in a group, rather than in how neurons interact in a single brain? On pages 422 and 421 of this issue, Rose et al. (1) and Báez-Mendoza et al. (2) show that brains of bats and rhesus macaques track group interactions by, respectively, coordinating their activity and representing the group in single neuron activity.

Group living enables species to come up with solutions that each individual would not have accomplished alone. These include reducing predation or accomplishing substantial tasks, such as ants' nests or human cities. These exceptional endeavors emerge from individuals' social interactions, which are sometimes as simple as copying local neighbors and sometimes more elaborate, involving memory of others and signaling (3-5). The study of social interactions in neuroscience has been carried out by recording neural activity in the brains of two individuals simultaneously and led to the discovery that brains in a group of two can couple their activity (6). Brain-to-brain coupling has been reported in various social interactions, including communication, cooperation, competition, and learning. It has been shown to affect neural activity at different spatiotemporal scales (including neuronal firing, neural oscillations, and hemodynamic responses) and in different brain regions. Further, the temporally coherent link between the activity of two brains was either synchronous or asynchronous and directed from one brain to the other. Brain-to-brain coupling has been demonstrated with increasingly sophisticated analysis methods and in different species, including bats, mice, and monkeys (7-9).

Yet it is only when multiple social interactions are coupled together that the power of a collective dynamic emerges. The change of scale from studying one social interaction between two individuals to studying collective social interactions within a group of individuals must go hand in hand with innovative methods. In human studies, the strategy has been to choose devices that are easy to use in a real environment, such as a classroom or a music studio, while being well coordinated to simultaneously record brain activity within the group in real time (10-12). In their investigations with Egyptian fruit bats and rhesus macaques, Rose et al. and Báez-Mendoza et al., respectively, took another direction. They did not want to trade off the resolution of single-neuron activity. They focused on the frontal cortex, which is involved in making and pondering decisions; the medial region is a hub of social cognition. They recorded from close, but not homologous, brain areas in the two species, allowing them to identify the activity of neurons in the frontal cortex that is sensitive to the identity of the individuals that the bats and monkeys interacted with. This means that interactions were tailored to the specific interacting partner (see the figure). In bats, this neuronal sensitivity was even more precise for close partners within the group than for distant partners, which were less well differentiated by neurons. In monkeys, dorsomedial prefrontal cortex neurons were active only during interactions; looking at the partner did not activate these neurons. When the interacting partner was a monkey plush puppet or a bat playback sound, these neurons stopped being active altogether. This contrasts with neurons representing peers in the nonhuman primate temporal cortex that are active when their peers' faces are presented as pictures (13). In the frontal cortex, these neurons appear to be tuned for possible action from peers, representing them as communication partners, competitors, and collaborators.

When socially interacting, the brains of two bats will couple their activity by synchronizing their frontal cortex neural oscillations as well as neuronal spiking activity (7). However, Rose et al. found that when a bat emits a call, interbrain 
coupling applies to all listening bats. Calling not only widely distributes information within the group but also induces collective brain coupling. By providing shared auditory stimulation to all brains at once, it could be that a communication call is not more specific than a gong or a bell ring in inducing collective brain coupling. However, communicative calls were found to be very specific in how they affected collective brain coupling within the bat group. The authors trained bats to emit vocalization to receive food from humans in the presence of other bats, but these trained calls did not elicit the same collective brain activity coupling as real communication. The caller's brain was no longer influenced by activity from the listener's brain when emitting trained calls. Conversely, real communication relied on bidirectional interbrain coupling, where the caller's brain accounts for the listener's brain before they send a signal.

Should research now focus on groups? In social insects, such as ants or bees, individuals are unable to represent the collective outcome of their group. Conversely, individual humans and most nonhuman primates can recapitulate part of the knowledge of the group, including the structure of their society and its culture, and use it for their own purpose $(5,14)$. This makes it interesting to study how a single brain accomplishes this advanced cognitive ability. Báez-Mendoza et al. show how a group of rhesus macaques derive economic laws from their trading interactions and represent this knowledge in their brain to guide future decisions. The authors used a simple, yet highly controlled, task that taps into the foundation of economic trade in humans: giving, receiving, and reciprocating (15). Monkeys sitting at a turntable were sequentially distributing pieces of apple to each other. Monkeys chose strategically to reciprocate gifts of apple to monkeys that gave them apple and to retaliate against those who did not give to them. In their decisions, monkeys not only considered the last gift received but also the history of giving and even the reputation of individuals to give to guide their own behavior. Such behavior was absent when monkeys could reward plush monkey totems.

During the economic trade, Báez-Mendoza et al. found that single-neuron activity from the dorsomedial prefrontal cortex reflects reward history in the group, monitoring the social outcomes of other monkeys' choices. Based on neuron activity, they were able to decode past interactions. Future reciprocation and retaliation were also predicted, even before the monkey decided to give a piece of apple. The neuronal activity that they discovered not only reflected monkeys' behavior but also was necessary for performing reciprocation behavior. When neuronal activity of the medial prefrontal cortex was temporarily disrupted - through reversible microstimulation — just before a monkey made their decision, reciprocation was diminished.

The findings of Rose et al. and Báez-Mendoza et al. are major steps in identifying the neural mechanisms for maneuvering in complex social structures, including those for interacting with others and for knowing the specific identity of the interactors and the history of those interactions. It remains unclear how neurons from the same area can be involved in these different processes at the same time. It will be important to understand which neurons' activity contributes to synchronization versus encoding social knowledge. Are there separate populations of neurons, or are the same neurons doing both tasks? The resolution with which brain activity is probed will be key to answering this question. These studies also emphasize that in the future, it will be necessary to navigate between two levels of investigation to fully grasp social intelligence: that of the group of brains and that of the individual brain. Further research should assess additional dynamical properties of animal and human collectives, including changes that may result from virtual collectives on the internet. $J$

REFERENCES AND NOTES

1. M. C. Rose et al., Science 374, eaba9584 (2021).

2. R. Báez-Mendoza et al., Science 374, eabb4149 (2021).

3. I. Couzin, Nature 445, 715 (2007).

4. A. Pentland, Adapt. Behav. 15, 189 (2007).

5. J. Krause et al., Trends Ecol. Evol. 25, 28 (2010).

6. G. Dumas et al., PLOS ONE 5, e12166 (2010).

7. W. Zhang, M. M. Yartsev, Cell 178, 413 (2019)

8. L. Kingsbury et al., Cell 178, 429 (2019).

9. K. M. Gilbert et al., bioRxiv 10.1101/2021.02.08.430294 (2021)

10. S. Dikker et al., Curr. Biol. 27, 1375 (2017).

11. V. Müller et al., Ann. N. Y. Acad. Sci. 1423, 198 (2018).

12. J. Yang et al., Nat. Neurosci. 23, 754 (2020).

13. S. M. Landi et al., Science 373, 581 (2021).

14. D. Cheney et al., Science 234, 1361 (1986).

15. M. Mauss, The Gift: Forms and Functions of Exchange in Archaic Societies (Cohen \& West, 1966).

ACKNOWLEDGMENTS

Funding is from the Agence Nationale de la Recherche (ANR-21-CE37-0016-02, ANR-19-CE37-0002-01, and ANR-10-IAIHU-06) to J.S. 